\title{
Mechano-Electric Feedbacks in a New Model of the Excitation-Contraction Coupling in Human Cardiomyocytes
}

\author{
Nathalie Balakina-Vikulova ${ }^{1,2}$, Olga Solovyova ${ }^{1,2}$, Alexander Panfilov ${ }^{2,3}$, Leonid Katsnelson ${ }^{1,2}$ \\ ${ }^{1}$ Institute of Immunology and Physiology of the Ural Branch of the Russian Academy of Sciences, \\ Ekaterinburg, Russia \\ ${ }^{2}$ Ural Federal University, Ekaterinburg, Russia \\ ${ }^{3}$ Ghent University, Ghent, Belgium
}

\begin{abstract}
The study is aimed to develop a new human cardiomyocyte model, which describes electromechanical coupling and mechano-electric feedbacks. The combined electromechanical model $(T P+M)$ links the TP06 electrophysiological model of the human cardiomyocyte with our earlier developed model of the myocardium mechanical activity and its calcium regulation.

In the $T P+M$ model, we tried to maintain principal features of calcium transients and action potentials during the twitches typical for the human cardiomyocytes.

The developed TP $+M$ model allows simulating several basic classic phenomena such as load-dependent relaxation and length-dependence of isometric twitches and respective changes in action potential duration. We have also simulated some age-dependent changes in the electrical and mechanical activity in the human cardiomyocytes.
\end{abstract}

\section{Introduction}

Development of personalized mathematical models of the human heart has become a challenge to increase the efficiency of medical practice. Three-dimensional multiscale modeling requires careful development of cellular level models to account for intracellular mechanisms underlying the coupling between heart excitability and its pump function.

The experimental data on the electromechanical activity of the human cardiomyocyte are very limited. Mathematical models allow integrating the available scarce and scattered experimental data obtained on healthy and pathological human myocardium into the whole picture, and adapting some data obtained on experimental animals.

Several reputed models of the human cardiomyocytes describe electrophysiological function of the cells [1-5]. They are widely used for $2 \mathrm{D}$ and $3 \mathrm{D}$ modeling to study mechanisms of cardiac arrhythmia in human [6]. Two available models of the excitation-contraction coupling in the human cardiomyocytes [7,8] combine the TP06 electrophysiological model [4] with the Rice [9] and Negroni-Lascano [10] models of the mechanical activity of the cardiac muscle, respectively. However, the referred studies do not provide an analysis of mechano-electric feedbacks (MEF) at the cellular level.

Meanwhile, intracellular MEF underlies an important pathway of myocardial auto-regulation adjusting its contractile function to the mechanical loading and contributing to the mechanical interactions between heterogeneous cardiomyocytes in the walls of the heart ventricles. In experiments on cardiac muscle preparations, MEF is revealed in several basic phenomena, including length-dependence of contractions and load-dependent relaxation. These and similar experiments were performed many times on papillary muscles and trabecules of various animal species (e.g. [11]). However, for obvious reasons, it is difficult to obtain similar data in human preparations. Therefore, just mathematical modeling is to be in high demand for predicting possible effects of MEF in human cardiomyocytes. We developed here such mathematical model and apply it, in particular, to simulate mechanodependent activity of adult and aged human cardiomyocytes.

The cross-links between the electrical and mechanical activity in the new model are reproduced due to accounting for cooperative mechanisms of $\mathrm{Ca}^{2+}$ activation of myofilament regulatory units in the cell.

\section{Methods}

\subsection{Mathematical model}

We developed a combined electromechanical model $(\mathrm{TP}+\mathrm{M})$ based on the TP06 electrophysiological model of the human cardiomyocyte [4] and our model of the myocardium mechanical activity and calcium handling [12].

The model of mechanical activity has been developed 
earlier as a component in the Ekaterinburg-Oxford (EO) electromechanical model developed for various laboratory animal cardiomyocytes [13]. Description of the cooperativity of regulatory and contractile proteins is a key feature of the EO model allowing us to explain and reproduce a wide range of mechano-calcium and mechanoelectric feedbacks.

The TP+M model combines the electrical and mechanical equations via the equations for intracellular calcium kinetics, which is intrinsic but differently detailed part of the cellular electromechanical models. For this linkage, we had to introduce thorough description of the calcium - troponin $\mathrm{C}(\mathrm{CaTnC})$ kinetics, because it is a key mechanism of the myocardium contraction activation, whereas in the TP06 model this kinetics was implied only as a part of a simplified single intracellular calcium buffer. The following equation inherited from the EO model is used in the TP+M model for the CaTnC concentration time course:

$$
\begin{aligned}
\frac{d[\operatorname{CaTnC}]}{d t}=k_{\text {on }} & \cdot\left([\operatorname{Tn} C]_{\text {tot }}-[\operatorname{CaTnC}]\right) \cdot\left[\mathrm{Ca}^{2+}\right]_{i}- \\
& -k_{\text {off }}([\operatorname{CaTnC}], N) \cdot[\operatorname{CaTnC}]
\end{aligned}
$$

where $[\mathrm{TnC}]_{\text {tot }}-$ total concentration of troponin $\mathrm{C}$ in the cell, $\left[\mathrm{Ca}^{2+}\right]_{\mathrm{i}}-$ free intracellular calcium concentration, $\mathrm{N}-$ fraction of force-generating crossbridges, $\mathrm{k}_{\mathrm{on}}$ and $\mathrm{k}_{\text {off }}([\mathrm{CaTnC}], \mathrm{N})$ are on- and off-rate "constants" of the CaTnC complex formation. Nonlinear dependence of the off-rate on [CaTnC], $\mathrm{N}$ and sarcomere length accounts for the cooperative mechanisms of calcium activation of thin myofilaments. Detailed formulations of cooperativity mechanisms have been done and justified earlier [12]. These mechanisms are the keys to the mechano-calcium feedbacks and consequently to the mechano-electric feedbacks. Thus, the generalized buffer described reductively by a quasi-stationary algebraic equation in the TP06 model we replaced by differential equations for cytosol $\mathrm{Ca}^{2+}$ buffers including Eq. (1) for the CaTnC.

To keep the species-specificity of the TP06 model we maintained qualitative and even quantitative features of the $\mathrm{Ca}^{2+}$ transient (i.e. time course of the changes in the cytosol $\mathrm{Ca}^{2+}$ concentration) during the contraction-relaxation cycle by fitting parameters of $\mathrm{Ca}^{2+}$ kinetics. It was especially necessary as the shape and duration of the $\mathrm{Ca}^{2+}$ transient in the TP06 model determine the shape and duration of the action potential (AP) specific just for the human cardiomyocytes. To simulate time characteristics of the isometric twitch of the human cardiomyocyte obtained experimentally [14] we fitted some parameters of the mechanical component, as well.

Significant challenge of the combining of the two models was to obtain mechano-dependence of the AP duration. Analysis of model parameters showed that the sodium-calcium exchange current $\left(\mathrm{i}_{\mathrm{NaCa}}\right)$, which plays an important role in MEF in the cardiomyocyte, was not sufficiently sensitive to changes in the concentration of intracellular calcium in the TP06 model. A parametrical tuning of the $\mathrm{i}_{\mathrm{NaCa}}$ within the combined $\mathrm{TP}+\mathrm{M}$ model allowed us to obtain a good mechanical dependence of the AP (see Results).

\subsection{Simulation of aged cardiomyocyte}

Data on the age-related intracellular changes in the myocardium is widely reported (e.g. [15]). The integrative effects of these changes on the electrical and mechanical activity in cardiomyocytes are still insufficiently explored [16].

Here, we used the TP+M model to simulate effects of age-related changes in the sarcoplasmic reticulum (SR) ATPase activity [17] on the excitation-contraction coupling in the human cardiomyocytes. To simulate the slowing down of $\mathrm{Ca}^{2+}$ uptake in ageing myocardium, we decreased maximal velocity of the SR pump by $50 \%$ as compared to the reference value.

All results are shown for the steady-state contractions at a pacing rate of $1 \mathrm{~Hz}$.

\section{Results}

\subsection{Load dependence of the electromechanical activity}

The main experimental modes used to study mechanical activity in myocardium are isometric and isotonic twitches at different sarcomere lengths (preload) and mechanical loads (afterload). Isometric contractions develop at a fixed length of the preparation during the cycle. During the isotonic phase of the afterloaded twitch, a myocardial preparation shortens and lengthens under a fixed constant load. Figure 1 illustrates the mechanical and electrical activity and $\mathrm{Ca}^{2+}$ transients in the cardiomyocyte during isometric (bold lines) and afterloaded twitches under different afterloads (decreased from dark to light grey lines) in the TP+M model. The duration of afterloaded twitches shortens with a decrease in afterload. The endsystolic shortening of the virtual preparation increases along with an increase in the velocity of deformation under the afterload decrease.

The mechanisms of mechano-calcium and mechanoelectric feedbacks reveal themselves in the prolongation of $\mathrm{Ca}^{2+}$ transient and AP with decreased afterload. The $\mathrm{Ca}^{2+}$ transient decay slows down via cooperative dependence of CaTnC kinetics on fraction of force-generated crossbridges. The slower $\left[\mathrm{Ca}^{2+}\right]_{\mathrm{i}}$ decay corresponds to the longer AP duration. 

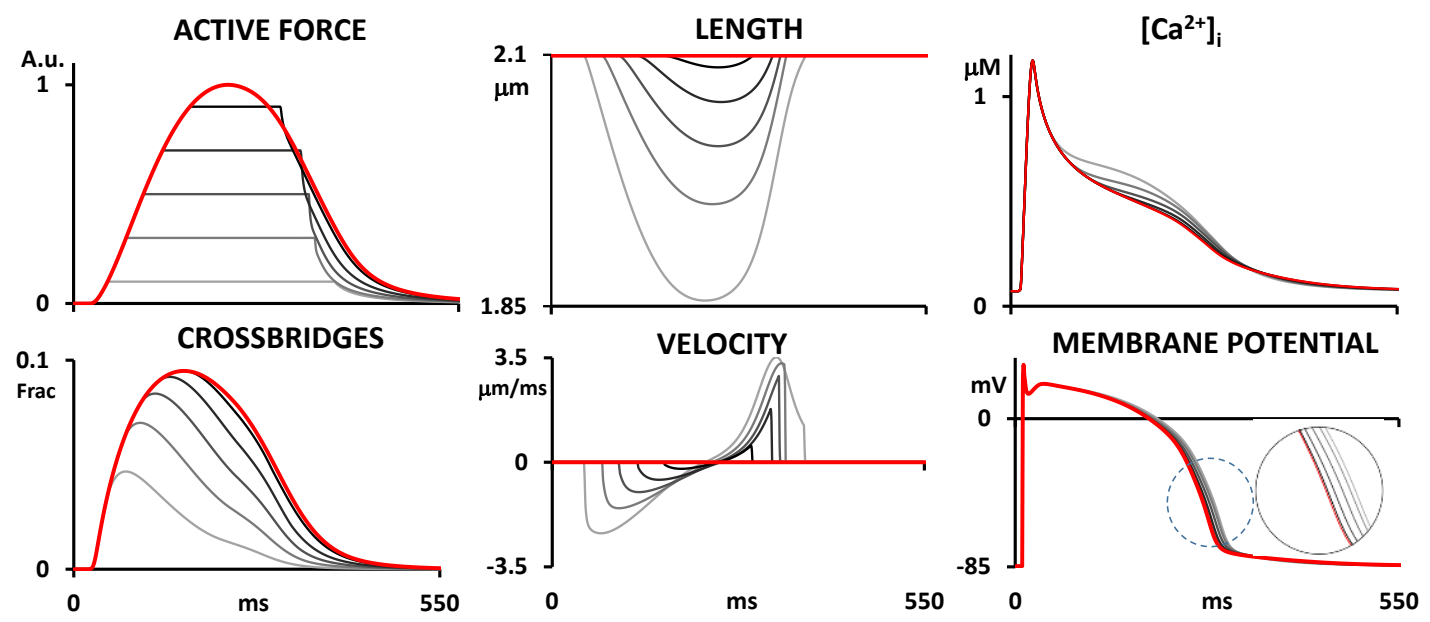

Figure 1. Afterload-dependent twitches. Bold (red) lines show the signals during isometric twitch. Lines from dark to light grey show signals during isotonic contractions under decreasing afterloads. Force values are normalized to the peak isometric value. The insert shows scaled-up differences in the AP duration under different afterloads.

\subsection{Isometric twitches at different lengths}
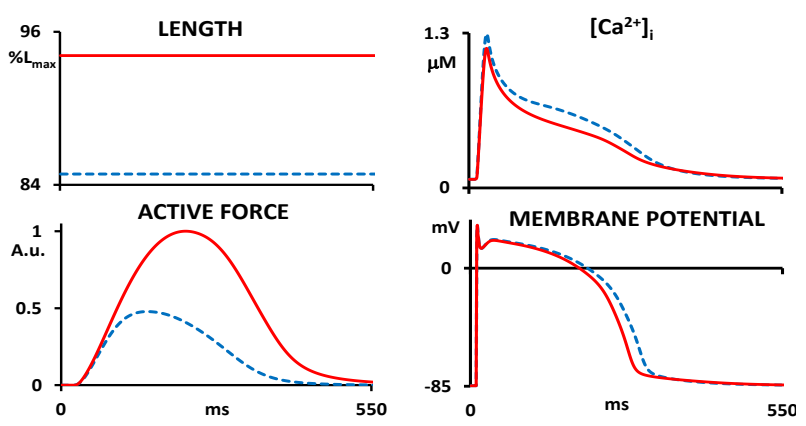

Figure 2. Preload-dependent isometric twitches. Solid (red) lines are for the bigger initial cell length as compared to the dashed (blue) lines. Force values are normalized to the peak value of the more elongated cell.

The TP+M model reproduces the dependence of the electromechanical activity of the cell on the initial sarcomere length (preload). Figure 2 shows the dynamic changes in the force, free cytosolic $\mathrm{Ca}^{2+}$ and transmembrane potential in the cell during isometric twitch at two lengths: $0.85 L_{\max }$ and $0.95 L_{\max }$ (where $L_{\max }$ is the length at which the maximum isometric force is generated). The model demonstrates significant increase in the time to and in the peak of isometric force at the higher length. Simulated $\mathrm{Ca}^{2+}$ transients during isometric contractions show a slightly reduced peak and shorter duration in the stretched cell. AP at the longer cell length is also shorter reflecting MEF mechanisms accounted for in the $\mathrm{TP}+\mathrm{M}$ model. The $\mathrm{TP}+\mathrm{M}$ model also produces mechano-calcium and mechano-electric feedback effects for various protocols of quick deformations (stretch and release) during the isometric twitch (unshown here).

\subsection{Simulation of the aging}

It is well documented that one of the essential change in excitation-contraction coupling with aging is a reduction in the SR $\mathrm{Ca}^{2+}$ pump activity [17]. Here, we tested consequences of a 50\% reduction in the maximal SR pump velocity (rate-constant) in the $\mathrm{TP}+\mathrm{M}$ model (Fig. 3). The two-fold decrease in this parameter resulted in a 30\% decrease in the SR diastolic level and considerable decrease in the peak of the $\mathrm{Ca}^{2+}$ transient and an increase in the time to peak (Fig. $3,\left[\mathrm{Ca}^{2+}\right]_{\mathrm{i}}$ ). The total amount of $\mathrm{Ca}^{2+}$ released from SR reduces by $50 \%$ as compared to the reference model. This was accompanied with an increase in the amount of $\mathrm{Ca}^{2+}$ entering the cell during excitation via $i_{\mathrm{CaL}}$ and $\mathrm{i}_{\mathrm{NaCa}}$ by $10 \%$. Particularly, the reverse-mode of $\mathrm{i}_{\mathrm{NaCa}}$ brings $15 \%$ more $\mathrm{Ca}^{2+}$ to the cell as compared to the reference model (Fig. 3). An increase in external entering $\mathrm{Ca}^{2+}$ partially compensates $\mathrm{Ca}^{2+}$ unloading in the SR.

A decrease in the bulk intracellular $\mathrm{Ca}^{2+}$ resulted in the significant decrease in the active force produced by the ageing model (Fig. 3). The changes in the $\mathrm{Ca}^{2+}$ handling affected AP morphology and duration (Fig. 3, membrane potential). The 'spike-and-dome' phase became less pronounced, and AP duration increased. Both a decrease in the force production and prolongation of the AP produced by the model with reduced SR $\mathrm{Ca}^{2+}$ pump activity reflect these most distinctive signs of the agerelated changes in the heart. 

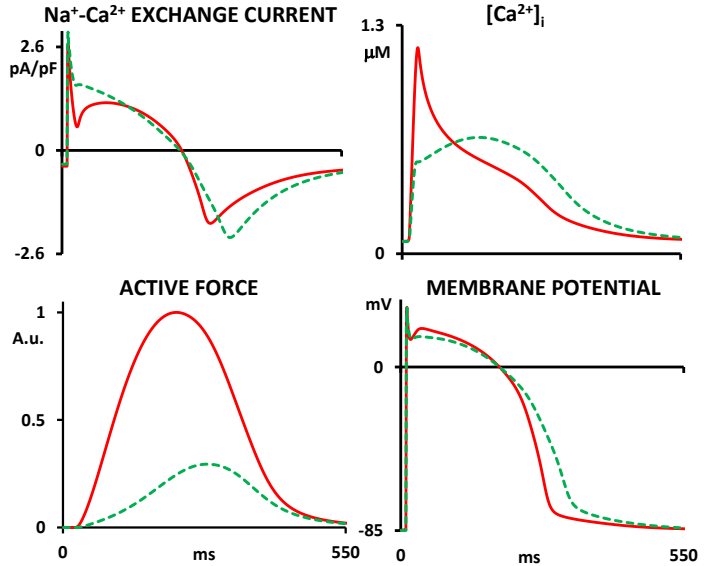

Figure 3. Age-related changes in the cardiomyocyte activity. Solid (red) lines are the signals produced by the reference model; dashed (green) lines are produced at the two-fold decrease in rate-constant of the SR $\mathrm{Ca}^{2+}$ pump. Force is normalized to the control peak.

\section{Conclusions}

The developed $\mathrm{TP}+\mathrm{M}$ model simulates the direct and inverse relations between electrical and mechanical phenomena in the human cardiomyocytes thanks to the mechanisms of cooperativity. The TP+M model may be further incorporated in the multiscale models of multicellular myocardium up to that of the whole ventricles and used for systematical computational assessment of the MEF contribution to the regulation of mechanical and electrical functions in the human hearts.

\section{Acknowledgements}

The work was carried out within the framework of the IIP UrB RAS themes (Nos. AAAA-A18-118020590031-8, AAAA-A18-118020590134-6) and was supported by Act 211 Government of the Russian Federation, contract № 02.A03.21.0006, and by RFBR (18-01-00059 - single cell modeling; 18-015-00368 - ageing simulation).

\section{References}

[1] Priebe L, Beuckelmann DJ. Simulation study of cellular electric properties in heart failure. Circ Res 1998;82(11):1206-23.

[2] Grandi E, Pasqualini FS, Bers DM. A novel computational model of the human ventricular action potential and $\mathrm{Ca}$ transient. $\mathrm{J}$ Mol Cell Cardiol 2010;48(1):112-21.

[3] ten Tusscher KH, Noble D, Noble PJ, Panfilov AV. A model for human ventricular tissue. Am J Physiol Heart Circ Physiol 2004;286(4):H1573-89.

[4] ten Tusscher KH, Panfilov AV. Alternans and spiral breakup in a human ventricular tissue model. Am J Physiol Heart Circ Physiol 2006;291(3):H1088-100.
[5] O'Hara T, Virag L, Varro A, Rudy Y. Simulation of the undiseased human cardiac ventricular action potential: model formulation and experimental validation. PLoS Comput Biol 2011;7(5):e1002061.

[6] Trayanova NA, Chang KC. How computer simulations of the human heart can improve anti-arrhythmia therapy. J Physiol 2016;594(9):2483-502.

[7] De Oliveira B, Rocha B, Barra L, Toledo E, Sundnes J, Weber dos Santos R. Effects of deformation on transmural dispersion of repolarization using in silico models of human left ventricular wedge. Int $\mathbf{J}$ Numer Method Biomed Eng 2013;29(12):1323-37.

[8] Lascano EC, Said M, Vittone L, Mattiazzi A, MundinaWeilenmann C, Negroni JA. Role of CaMKII in post acidosis arrhythmias: a simulation study using a human myocyte model. J Mol Cell Cardiol 2013;60:172-83.

[9] Rice JJ, Wang F, Bers DM, De Tombe PP. Approximate model of cooperative activation and crossbridge cycling in cardiac muscle using ordinary differential equations. Biophys J 2008;95(5):2368-90.

[10] Negroni JA, Lascano EC. Simulation of steady state and transient cardiac muscle response experiments with a Huxley-based contraction model. J Mol Cell Cardiol 2008;45(2):300-12.

[11] Kaufmann R, Lab M, Hennekes R, Krause H. Feedback interaction of mechanical and electrical events in the isolated mammalian ventricular myocardium (cat papillary muscle). Pflügers Archiv 1971;324(2):100-23.

[12] Sulman T, Katsnelson LB, Solovyova O, Markhasin VS. Mathematical modeling of mechanically modulated rhythm disturbances in homogeneous and heterogeneous myocardium with attenuated activity of $\mathrm{Na}^{+}-\mathrm{K}^{+}$pump. Bull Math Biol 2008;70(3):910-49.

[13] Solovyova O, Vikulova N, Katsnelson LB, Markhasin VS, Noble P, Garny A, et al. Mechanical interaction of heterogeneous cardiac muscle segments in silico: effects on $\mathrm{Ca}^{2+}$ handling and action potential. Int $\mathrm{J}$ Bifurcat Chaos 2003; 13(12):3757-82.

[14] Janssen PM, Lehnart SE, Prestle J, Hasenfuss G. Preservation of contractile characteristics of human myocardium in multi-day cell culture. J Mol Cell Cardiol 1999;31(8):1419-27.

[15] Paneni F, Diaz Canestro C, Libby P, Luscher TF, Camici GG. The Aging Cardiovascular System: Understanding It at the Cellular and Clinical Levels. J Am Coll Cardiol 2017;69(15):1952-67.

[16] Nakou ES, Parthenakis FI, Kallergis EM, Marketou ME, Nakos KS, Vardas PE. Healthy aging and myocardium: A complicated process with various effects in cardiac structure and physiology. Int J Cardiol 2016;209:167-75.

[17] $\mathrm{Xu} \mathrm{A}$, Narayanan N. Effects of aging on sarcoplasmic reticulum $\mathrm{Ca}^{2+}$-cycling proteins and their phosphorylation in rat myocardium. Am $\mathrm{J}$ Physiol 1998;275(6 Pt 2):H2087-94.

Address for correspondence.

Nathalie Balakina-Vikulova (n.vikulova@iip.uran.ru)

Institute of Immunology and Physiology of the Ural Branch of the Russian Academy of Sciences, Pervomayskaya st., 106, 620041, Ekaterinburg, Russian Federation 